\title{
PARLAMENTARISCHE DEMOKRATIE IN BANGLADESH EINE ANALYSE DES VERFASSUNGSZUSTANDS
}

\author{
Von Horst Hartmann
}

\section{Hintergrund}

Es gibt nur einige Entwicklungsländer, die für die Institutionalisierung eines demokratischen Verfassungsstaates optiert haben und in der Entscheidung für diese Verfassungsform die Krönung ihrer Unabhängigkeitsbewegung sahen. Zu diesen Ländern gehört Bangladesh, das als neues Staatsgebilde am 16. Dezember 1971 entstand.

Die Geburt von Bangladesh war ein besonderes Phänomen: Zum ersten Mal war in einem Entwicklungsland gegen „internen Kolonismus“ rebelliert worden; Bangladesh hatte sich von den Fesseln der westpakistanischen Oberherrschaft befreit. Mit Aufmerksamkeit und Sympathie verfolgte die Weltöffentlichkeit den Freiheitskampf der Bevölkerung Ostpakistans. In diese Welle von Anteilnahme und Faszination mischten sich Zweifel hinsichtlich der Lebensfähigkeit des neuen Staates. Weitverbreitete Armut, Analphabetentum und Arbeitslosigkeit bilden eine schlechte Grundlage für politische Stabilität.

Das Porträt eines der ärmsten Entwicklungsländer wird durch folgende dominierende Züge charakterisiert:

- Auf 143000 qkm Landfläche leben nach dem Zensus von 1973 rd. 72 Mill. Menschen. Das entspricht einer Bevölkerungsdichte von etwa 500 Menschen pro $\mathrm{qkm}$. Bei einem anhaltenden Bevölkerungswachstum von 3\% wird sich dieser Bevölkerungsdruck zu einer Bevölkerungsexplosion entwickeln. Bei einer landwirtschaftlichen Nutzfläche von 9,4 Mill. ha stehen nur 1,25 qm, noch nicht einmal die Größe eines Schrebergartens, pro Kopf zur Verfügung1.

- Die industriellen Voraussetzungen sind ungünstig. Mit Ausnahme von Gas und einigen ökonomisch unrentabelen Kohle- und Kalkvorkommen besitzt Bangladesh keinerlei Rohstoffe, die für den Aufbau einer Industrie genutzt werden könnten. Es kommt hinzu, daß die industrielle Produktion während der kriegerischen Auseinandersetzungen fast völlig zum Stillstand gekommen war. Nach Schätzungen der Planungskommission waren Produktionsanlagen und Vorratslager im Wert von 220 Mill. Taka (nach festgesetztem Kurs erhält man für 1,- DM 3,- Taka) beschädigt und zerstört worden². Die Abreise westpalkistanischer Manager und Facharbeiter hinterließ auf dem Arbeitsmarkt ein noch immer nicht geschlossenes Vakuum ${ }^{3}$.

* Vergleiche: Sen, The Constitution of Bangladesh and a Short Constitutional History, 7 VRU (1974), S. 257.

1 Vgl.: Bundesstelle für Außenhandelsinformationen (Hrsg.), Marktinformationen - Bangladesh, Wirtschaftslage 1972/73, Köln, April 1974, S. 1 ff. - In diesem Bericht werden die Ergebnisse einer Untersuchung wiedergegeben, die 1973 im Auftrag des Ostasiatischen Vereins e. V., Hamburg, durchgeführt wurde.

2 Interview des Verf. mit Prof. Rohman Sobhan, Mitglied der Planungskommission, Dacca, 13. 8. 1974. Prof. Sobhan ist im Oktober 1974 aus Protest gegen die Wirtschaftspolitik der Regierung zurückgetre-

3 Vgl.: Dilip Mukerjee, Challenges Facing Bangladesh, II - Fallout From Repatriation, in: The Times of India, Delhi, 24. 11. 1973. 
- Die natürlichen Bedingungen sind schlecht. Kardinalproblem ist die Inangriffnahme von Flußregulierungen und Flutkontrollprogrammen. Das Delta des Ganges-Brahmaputra ist den Launen dieser beiden mächtigen Ströme hilflos ausgesetzt. In der Monsunzeit überschwemmen die Wassermengen - wie auch 1974 - etwa die Hälfte der kultivierten Fläche ${ }^{4}$ und vernichten alles, was sich ihnen in den Weg stellt.

- Acht von zehn Bengalen sind Analphabeten. Der Kinderreichtum sorgt dafür, daß die Rolle der Frau auf Familie und Heim beschränkt bleibt.

Dieser Ausgangslage entspricht der wirtschaftliche Entwicklungsstand. Ein ProKopf-Einkommen von 192,- DM pro Jahr $(1972 / 73)^{5}$ ist Indiz dafür, daß die Mehrheit der Bevölkerung unter dem Existenzminimum dahinvegetiert.

Der landwirtschaftliche Sektor dominiert mit einem Beitrag von etwa $55 \%$ zum BSP in der Volkswirtschaft. Das technische Niveau, vor allem die Produktionsmethoden und die ha-Erträge (Reis, Weizen, Zuckerrohr) zählen zu den niedrigsten der Erde. $75 \%$ der Gesamtbevölkerung sind direkt in der Landwirtschaft beschäftigt, während $80 \%$ in ländlichen Gebieten leben. Die Siedlungsstruktur ist äußerst zersplittert. Die Mehrzahl der 65000 Dörfer ist isoliert, nur 2000 sind an das Straßennetz angeschlossen und nur etwa 200 sind elektrifiziert.

Obwohl im Gegensatz zu Indien feudalistische Züge nahezu gänzlich fehlen, ist die Bodenverteilung sehr ungleich. Eine ländliche Elite, die etwa $15 \%$ der Bauernschaft umfaßt, besitzt ca. 50\% der bebauten Fläche. Dieser besitzenden Schicht stehen $15 \%$ völlig landlose und $12 \%$ quasi landlose (weniger als 0,5 acre Landbesitz) Bauern gegenüber ${ }^{6}$.

Ein großer Teil der landwirtschaftlichen Produktion dient dem Eigenbedarf. Aus den erwirtschafteten Überschüssen werden Schulden abgetragen und die dringendsten Bedürfnisse nach Gebrauchsartikeln befriedigt. Die kaufkräftige Nachfrage ist minimal, so daß der Markt kaum Anreiz zu einer Ausdehnung der Produktion bietet. Das landlose Proletariat und die städtischen Arbeitslosen haben zwar großen Bedarf, verfügen aber über keine nennenswerte Kaufkraft.

Trotz des von der Regierung proklamierten "Sozialismus“ findet sich im Plan für 1973/74 kein Hinweis auf Landreform oder Verstaatlichung des Grundbesitzes.

Zur Furcht um die wirtschaftliche Lebensfähigkeit des Landes gesellte sich die Sorge um die Bewahrung seiner Souveränität. Bangladesh hat mit Indien, das bei seiner Entstehung eine entscheidende Rolle spielte, drei Grenzen gemeinsam. Das Land schien daher von vornherein als „Klient" des übermächtigen Nachbarn bestimmt zu sein.

Diese Sorge wurde durch die Tatsache verschärft, daß die administrativ-politische Struktur des Landes schwach ausgebildet war. Es fehlte an den einfachsten Voraussetzungen einer funktionsfähigen Verwaltung, zumal der Bürgerkrieg die rudimentär vorhandenen Ansätze einer Administration weitgehend vernichtet hatte.

Hinzu kam, daß die Awami-Liga, in deren Namen der Freiheitskampf geführt worden war, um diesem Legitimität zu verleihen - die Awami-Liga hatte 1970 in den Wahlen 167 von den 169 Sitzen, die auf Ostpakistan entfielen, gewonnen -,

\footnotetext{
4 Dazu siehe: Bernhard May, Die Entwicklung der Landwirtschaft in Bangladesh und Pakistan, in: Internationales Asienforum, Vol. 4, München 1973, S. 286.

5 Planning Commission, The First Five Year Plan 1973-78, Dacca, Nov. 1973, S. 15.

6 Vgl.: Bernhard May, a. a. O., S. 289 f. - Für weitere Einzelheiten siehe auch: Austin Robinson

Egl.: Bernhard May, a. a. O., S. 289 f. - Für weitere Einzelheiten siehe auch: Austin Robinson,
Economic Prospects of Bangladesh, London 1973, S. 21 f., u. Azizur Rahman Khan, The Economy of Bangladesh, London 1971 , S. $38 \mathrm{ff}$.
} 
die Nachbürgerkriegswirren ohne Sheikh Mujibur Rahman, ihren charismatischen Führer, überstehen mußte. Dieser wurde in Pakistan noch in Haft gehalten. Die Freilassung erfolgte im Januar 1972. In der Zwischenzeit mußte die Awami-Liga ihren Herrschaftsanspruch gegen verschiedene Fraktionen der Mukti Bahini verteidigen, in deren Händen vor allem die Führung des Freiheitskampfes in Ostpakistan gelegen hatte, während sich die Führung der Awami-Liga in Kalkutta im Exil befand und dort eine Regierung gebildet hatte ${ }^{7}$. Die Frage tauchte auf, ob die Awami-Liga unter diesen Umständen in der Lage sein würde, über das gesamte Gebiet von Bangladesh ihre Autorität zu errichten und Kontrolle auszuüben.

\section{Verfassung}

Trotz dieser mannigfachen Probleme ging der politische Aufbau des Landes zunächst verhältnismäßig zügig voran. Es gelang der provisorischen Regierung der Awami-Liga, Katastrophen (Hungersnöte, Epidemien) zu vermeiden, was freilich ohne die tatkräftige Hilfe der internationalen Völkergemeinschaft (insgesamt 1,3 Mrd. US \$) undenkbar gewesen wäre. Öfentliche Ordnung und Sicherheit konnten auf ein Mindestmaß stabilisiert werden. Das Flüchtlingsproblem wurde gelöst, wenn auch eine völlige Wiedereingliederung der zurückflutenden Menschenmassen in den Arbeitsprozeß nicht zu realisieren war. Der Wirtschaftsprozeß war nahezu zum Stillstand gekommen.

In den ersten Monaten nach seiner Haftentlassung regierte Sheikh Mujibur mit Hilfe seines damals noch unangefochtenen Charismas. Dieses nutzte er aber auch zum Aufbau einer politischen Struktur. Dabei schien das indische Modell der politischen Entwicklung als Vorbild zu dienen. Mit anderen Worten: Eine parlamentarische Demokratie mit einer dominierenden Partei und dem Bekenntnis zum Sozialismus und Säkularismus, was gleichbedeutend ist mit einem Verbot links- und rechtsextremistischer Opposition. Im Prinzip hatte die Awami-Liga das Verfassungsprogramm schon in ihrem Wahlmanifest von 1970 niedergelegt ${ }^{8}$.

Der Regierung unter Sheikh Mujibur ist es als großer Erfolg anzurechnen, daß bereits nach einem Jahr der Amtsübernahme die Verfassung verabschiedet werden konnte. Am 10. April 1972 war die Verfassungsgebende Versammlung für zwei Tage zusammengetreten und hatte unter dem Vorsitz des Justizministers Dr. Kamal Hossain ein 34 Mitglieder starkes Komitee mit dem Entwurf einer Verfassung beauftragt. Nach sechs Monaten lag dieser vor und wurde von der Verfassungsgebenden Versammlung, in der die Awami-Liga eine überwältigende Mehrheit besaß, in einer Rekordzeit verabschiedet. Am 16. Dezember 1972, genau ein Jahr nach der Geburt von Bangladesh, wurde die Verfassung mit nur wenigen Änderungen gegenüber dem Entwurf wirksam? .

Einer der Gründe für diese Eile lag in der Absicht der Regierung, ihre Legitimität auf eine Verfassungsgrundlage zu stellen. Als weiterer Grund muß hinter der Hast die Furcht vor einer Verfassungskontroverse gesehen werden. Der Keim dazu steckte in der wachsenden Unzufriedenheit breiter Bevölkerungsschichten.

7 Vgl.: Rounaq. Jahan, Bangladesh in 1972: Nation Building in a New State, in: Asian Survey, Vol. VIII, No. 2, California, February 1973, S. $199 \mathrm{ff}$

$8 \mathrm{Es}$ handelt sich um das berühmte Sechs-Punkte-Programm der Awami-Liga, das auch die programmatische Grundlage für die Verfassung abgegeben hat. Der Text ist abgedruckt in: Bangladesh Documents, Hrsg.: Ministry of External Affairs, New Delhi (o. J.), S. 66 ff. 
Die neue Verfassung ${ }^{10}$ enthält alle wesentlichen Elemente eines parlamentarischen Regierungssystems: Wahl der 300 Abgeordneten nach dem Prinzip der relativen Mehrheitswahl in Einer-Wahlkreisen, kollektive Verantwortung des Kabinetts vor dem Parlament mit dem Premierminister als "primus inter pares" und die Institution eines Präsidenten als formales Staatsoberhaupt. Das Recht zur Auflösung des Parlaments, das in Artikel 57, Absatz 2 der Verfassung vorgesehen ist, bildet in den Händen des Premierministers ein traditionelles Instrument, um die Reihen der Regierungspartei weitgehendst geschlossen zu halten. Einen zusätzlichen Damm zur Sicherung der Stabilität des Systems hat man durch die Aufnahme von Artikel 70 in die Verfassung errichtet. Dieser sieht vor, daß ein Abgeordneter seinen Sitz zur Verfügung stellen muß, wenn er aus einer Partei ausgetreten ist oder im Parlament gegen seine Partei gestimmt hat.

Ganz offensichtlich haben die schlechten Erfahrungen, die man in Indien mit dem Überwechseln von Abgeordneten von einer Partei zur anderen gemacht hat, als warnendes Beispiel gedient ${ }^{11}$. Durch diese Vorschrift, die - wie nicht anders zu erwarten - heftig umstritten war und in gewisser Beziehung noch abgemildert wurde, indem die ursprüngliche Klausel einer öffentlichen Notifizierung durch den Sprecher des Parlaments gestrichen wurde ${ }^{12}$, macht es praktisch unmöglich, daß die Awami-Liga durch die Abspaltung der Mehrheit ihrer Abgeordneten automatisch ihre Regierungsposition verlieren würde. In einem solchen Fall blieben die Alternativen der Bildung einer Minderheitenregierung oder der Ausschreibung von Neuwahlen.

Die Dominanz einer Partei ist damit verfassungsrechtlich abgestützt und bietet aus dieser Sicht geeignete Voraussetzungen für die Realisierung des indischen Modells. Es ist aber an dieser Stelle schon hervorzuheben, daß die Verfassungsentwicklung in anderen Bahnen zu verlaufen scheint.

Im zweiten Verfassungsteil werden als Grundprinzipien der Staatspolitik Nationalismus, Sozialismus, Demokratie und Säkularismus genannt. Das Bekenntnis zum Sozialismus wird untermauert durch die Verpflichtung des Staates zur Wirtschaftsplanung, Nationalisierung und Emanzipation der Massen. Insgesamt gesehen laufen diese Vorschriften auf eine Einschränkung der demokratischen Freiheiten und Rechte hinaus, wie man es in den westlichen Demokratien, aber auch in Indien nicht kennt.

\section{Parteien}

In den ersten allgemeinen Wahlen zum Parlament (Jayio Sangsad) stellten sich im März 1973 den Wählern eine Vielzahl von Parteien. Zwar war abzusehen, daß die Awami-Liga die absolute Mehrheit erringen würde. Ubberraschend aber war das Ausmaß des Sieges. Die Awami-Liga gewann in 291 der 300 Wahlkreise und vereinte auf sich 73 Prozent der abgegebenen Stimmen ${ }^{13}$.

9 The Bangladesh Observer, Dacca, 17. 12. 1972. Text auch in Verfassungstexte, Bangladesh. Beilage zu VRƯ 2/1973.

10 Vgl.: Verfassung der Volksrepublik Bangladesh, bearbeitet von Henning v. Wedel, als Beilage zu: Verfassung und Recht in Ubersee, Heft 3, Hamburg 1973.

11 Für Einzelheiten dazu vgl. vom Verf.: Political Parties in India, Meerut 1971, S. $41 \mathrm{ff}$.

$12 \mathrm{Vgl}$. Abdruck des ursprünglichen Textes in: The Bangladesh Observer, Dacca, 14. 10.1972.

13 Eine amtliche Veröffentlichung der Wahlergebnisse liegt nicht vor. Die Ergebnisse wurden zusammengestellt aus: The Bangladesh Observer, Dacca, 9.-10. 3. 1973. - In einem Wahlkreis fanden die Wahlen später statt. - Dazu und zu den weiteren Ausführungen vgl. auch: Swaroop Rani Dubey, Elections in Bangladesh, in: South Asian Studies, Vol. VIII, No. 2, Jaipur, July 1973, S. 62 ff. 
Parteien

Wahl resultate 1973

\begin{tabular}{lrrrr} 
& \multicolumn{2}{c}{ Sitze } & \multicolumn{2}{c}{ Stimmen } \\
& abs & \multicolumn{1}{c}{ rel. } & \multicolumn{2}{r}{ abs. in Mill. rel. } \\
\hline Awami League & 291 & 97.0 & 13.503 & 72.9 \\
National Awami Party (M) & 1 & 0.3 & 1.586 & 8.6 \\
National Awami Party (B) & - & - & 1.001 & 5.4 \\
Jatiyo Samajtantrik Dal (JSD) & 1 & 0.3 & 1.196 & 6.5 \\
Bangladesh Jatiyo League (BJL) & 1 & 0.3 & 52 & 0.3 \\
Bangla Jatiyo League & - & - & 53 & 0.3 \\
Communist Party of Bangladesh (CPB) & - & - & 47 & 0.3 \\
Communist Party of Bangladesh (Leninist) & - & - & 19 & 0.1 \\
Unabhängige & 5 & 2.0 & 915 & 4.9 \\
Splitterparteien & - & - & 120 & 0.8
\end{tabular}

Der Wahltriumph war vorwiegend Sheikh Mujibur persönlich zuzuschreiben. Seine Popularität war trotz des schwindenden Ansehens der Partei intakt geblieben.

Die hoffnungslos zersplitterte Opposition war mit ihren Klagen gegen die galoppierenden Preise und die Korruption mancher Regierungspolitiker während des Wahlkampfes bei den Wählern zwar auf Interesse gestoßen. Aber keine Oppositionsgruppe hatte personell oder programmatisch eine genügend attraktive Gegenposition aufgezeigt.

Enttäuschend war der Wahlausgang vor allem für die von Maulana Abdul Hamid Khan Bashani geführte National Awami Party (NAP), die nur im größten Distrikt des Landes Tangail mehr Resonanz bei den Wählern fand. Die Partei vertritt eine militante anti-indische Haltung und setzt sich für einen inhaltlich nicht genau definierten „islamischen Sozialismus ${ }^{14}$ “ ein. Damit aber gerät sie in die Nähe eines staatsgefährdenden Kommunalismus und in Gegensatz zum verfassungsrechtlich verankerten Säkularismus ${ }^{15}$.

Nach den Wahlen hat die NAP (B) durch weitere Abspaltungen noch mehr an Boden verloren. Es haben sich von ihr die extrem-linken Kräfte losgesagt und eine Splitterpartei gebildet, die sich als NAP (Requisitioned) betrachtet ${ }^{16}$. Reden macht von sich noch immer Bashani, der trotz seines greisen Alters politisch sehr aktiv ist und bei öffentlichen Auftritten die Massen zu emotionalisieren versteht.

Die von Prof. Muzzafar Ahmed geführte NAP, die 1968 aus einem Spaltungsprozeß der NAP (B) hervorgegangen ist, konnte auf sich zwar einen recht beachtlichen Stimmenanteil vereinigen, aber nur einen Abgeordneten in das Parlament schicken, weil ihr Wählerpotential zu sehr zersplittert ist. Die NAP (M) unterscheidet sich programmatisch nicht von der Awami-Liga und wird allgemein als ihr „B“-Team abgetan ${ }^{17}$.

\footnotetext{
14 Wie schwammig das Selbstverständnis der NAP (B) ist, zeigt die von Bashani stammende Abgrenzung des ,islamischen Sozialismus" vom "marxistischen Sozialismus ${ }^{\alpha}$, die im Bangladesh Observer abgedruckt wurde und lautet: "In Marxism nationalisation is made in the name of the State, while in Islamic

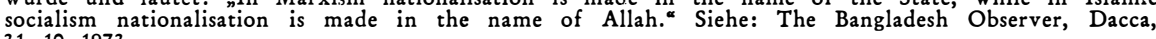
31. 10. 1973 .

15 Kennzeichnend dafür ist, daß Bashani Indien als ${ }_{n} \mathrm{Hindu}-\mathrm{Staat}{ }^{\star}$ bezeichnet.

$16 \mathrm{Vgl}$. Bericht in: Holiday, Dacca, 12. 5. 1974, S. 3.

$17 \mathrm{Im}$ Hinblick auf das Programm siehe: Aims, Objects \& Programme, Hrsg.: Bangladesh National Awami Party (M), Dacca 1972, S. $1 \mathrm{ff}$.
} 
Für viele Beobachter nicht überraschend kam das verhältnismäßig gute Abschneiden der Jatyo Samajtantrik Dal (JSD), was so viel wie „nationalsozialistische“ Partei heißt. Diese Organisation war erst im Oktober 1972 von dem früheren Studentenführer Abdur Rab und dem Freiheitskämpfer Major Jalil gegründet worden. Sie rekrutiert vor allem die Jugendlichen und enttäuschten Anhänger Mujibur Rahmans.

Diese „Nationalsozialisten“ sind gewiß keine Faschisten, sondern Sozialisten, die sich ihre Politik weder von Moskau noch von Peking diktieren lassen wollen. Obwohl die Partei in 236 Wahlkreisen Kandidaten aufgestellt hatte ${ }^{18}$, sieht sie ihr Ziel nicht im parlamentarischen Parteienkampf. Nur formal steht sie auf dem Boden der Demokratie ${ }^{19}$. Ihr geht es um den Aufbau einer kämpferischen Massenorganisation.

Die Bangla Jatiyo League (BJL) ist ohne Einfluß und ohne Programm. Sie besteht praktisch nur aus ihrem Präsidenten Atmur Rahman, der zeitweilig Zellengenosse von Mujibur Rahman war. Diese Tatsache macht ihn nach wie vor unpopulär und ließ ihn seinen Wahlkreis in Dacca gegen den Kandidaten der Awami-Liga gewinnen.

Insgesamt gesehen ist die parlamentarische Opposition tot. Das ist objektiv für eine Regierungspartei nicht von Vorteil. Es gibt innerparteilichen Fehden mehr Spielraum, weil die Gefahr einer Wachablösung nicht droht und damit auch der Zwang zur Kohäsion fehlt. Selbstgefälligkeit und Unentschlossenheit sind die Folge. Darüber hinaus wächst die Tendenz zur Bildung einer außerparlamentarischen Opposition, die sich in den Städten, vor allem bei der bengalischen Mittelklasse, regt.

Im Ggensatz zu Indien hat die Opposition im Parlament zu Dacca auch nicht die geringste Chance, ihre Ansichten wirksam zu artikulieren und die Regierung zu kontrollieren. In dieser Beziehung hat die Übertragung des indischen Modells auf Bangladesh ein anderes Ergebnis gebracht. Nicht auszuschließen ist, daß der Awami-Liga die Amputation der parlamentarischen Opposition ins Programm paßt, zumal bei Mujibur Rahman autokratische Züge nicht zu übersehen $\operatorname{sind}^{20}$. $\mathrm{Ihm}$ ist jeder suspekt, der in Opposition zur Regierung und damit auch zu ihm steht. Als „Vater der Nation" hält er sich für unfehlbar"21.

Auf diesem Hintergrund müssen auch der Rücktritt Mujibur Rahmans als Parteivorsitzender und auch die Neu- und Umverteilung der Ressorts gesehen werden, auf die wir weiter unten noch eingehen werden.

\section{Politische Lage}

Wenn sich trotz der Schaffung staatlicher Institutionen, die von der Mehrheit der Bevölkerung und der Opposition im Prinzip akzeptiert werden, und der Dominanz einer Partei die Awami-Liga-Regierung nicht tiefer in der Bevölkerung verwurzeln konnte, so liegt das an der zunehmend spürbar werdenden Diskrepanz zwischen Herrschaftsanspruch und wirtschaftlichem Mißerfolg sowie an der fehlenden Kraft,

18 The Bangladesh Observer, Dacca, 7. 3. 1973.

19 Interview des Verf. mit einem Parteimitglied, das nicht genannt werden möchte, Dacca.

20 Bekannt ist von ihm der nach den Wahlen von 1973 nach einer gewissen Befriedigung klingende Ausspruch: "There is now no opposition in the country. - Siehe dazu den Bericht von: Walter Schwarz, The loss of Bangladesh's innocence, in: The Guardian, Manchester, 12. 3. 1973.

21 Vgl. dazu den recht treffenden Bericht von: Carlos Widmann, Der Vater der Nation flieht ins Traumreich, in: Süddeutsche Zeitung, München, 2. Sept. 1974. 
neue Impulse zu geben. In dieser Beziehung brachte auch der erste Parteitag, den die Awami-Liga vom 18. 1. bis 20. 1.74 abhielt, wenig Neues. Anstelle der notwendig erscheinenden kritischen Überprüfung der Programme, versucht die Partei aus der Rückbesinnung auf ihre historische Rolle vor und während des Freiheitskampfes neues Selbstvertrauen zu schöpfen. Vermutlich war es der Parteiführung in erster Linie daran gelegen, im Zeichen einer schwelenden innenpolitischen Krise die Einheit der Partei zu demonstrieren.

Symptomatisch für den inneren Zustand der Awami-Liga war, daß sich die Erörterung organisatorischer Probleme auf Größe und Farbe der Parteiflagge konzentrierte $^{22}$. Das ist um so gravierender, als organisatorische Reformen überfällig sind und die bereits im September 1973 angekündigte „Reinigung“ der Partei noch immer im Raume steht, auch wenn man in letzter Zeit verstärkt korrupte Mitglieder aus der Partei ausgeschlossen hat.

Als wichtigstes Ergebnis des Parteitages war der Wechsel im Parteivorsitz, der von dem bisherigen Handelsminister A. H. Kamarussaman übernommen wurde, zu vermerken. Er löste Skeikh Mujibur Rahman ab, der dieses Parteiamt seit 1966 innehatte.

Diese Auflösung der Personalunion zwischen Regierungs- und Parteichef erfolgte in Übereinstimmung mit dem in der Parteisatzung niedergelegten Prinzip der Inkompatibilität beider Ämter. Ein Machtverlust dürfte sich daraus für den Sheikh nicht ergeben. Der neugewählte AL-Präsident ist ein alter Vertrauter - er war lange Jahre Generalsekretär der „All Pakistan Awami League"3“ und verfügt über keine nennenswerte eigene Hausmacht. Als weiteres Anzeichen dafür, daß Sheikh Mujibur Rahman in der Partei seinen dominierenden Einfluß weiterhin unverändert durchsetzen kann, ist der Umstand hervorzuheben, daß die alte Parteimannschaft mit einer Ausnahme (Ausscheiden des früheren Vizepräsidenten Korban Ali) im Amt bestätigt wurde.

Trotz aller Mißerfolge hat die Awami-Liga-Regierung offenbar den Glauben an den von ihr eingeschlagenen Weg (Nationalisierung der Industrie, der Banken und Versicherungen; Verstaatlichung des Außenhandels; Devisenbewirtschaftung und rigorose Importkontrolle) noch nicht verloren. Dabei kann die Regierung nicht mehr verheimlichen, daß die Wirtschaftslage schlechter geworden ist. Die Kennzeichen der Wirtschaftskrise sind: eine hohe Inflationsrate - die Planungskommission schätzt die Erhöhung des Lebenshaltungskostenindex im Jahre 1973 auf ca. 40 Prozent, doch dürfte der tatsächliche Kaufkraftschwund im Hinblick auf den Preisanstieg lebensnotwendiger Güter weitaus höher, bei ca. 60 Prozent liegen -, stagnierende Inlandsproduktion, wachsende Haushalts- und Zahlungsbilanzdefizite, Rückgang des Pro-Kopf-Einkommens im Vergleich zu 1969/70 um 20 Prozent ${ }^{24}$.

Die Folge war eine Anhäufung von Versorgungskrisen. Die Regierung wurde durch das permanente Krisenmanagement so in Anspruch genommen, daß ihr weder Zeit noch Kraft für eine Regierungsarbeit blieb. Eine zusätzliche Lähmung bei entwicklungspolitischen Grundsatzentscheidungen entsteht durch widerstreitende Eigeninteressen der bestehenden Gruppierungen innerhalb der Regierung. Die hem-

22 The Bangladesh Observer, Dacca, 19. 1. 1974.

23 Interview des Verf. mit A. H. Kamarussaman, Dacca, 3. 8. 1974.

24 Planning Commission, Economic Development in 1973-74 and Annual Plan for 1974-75, Dacca, July 1974, S. 1. 
menden Effekte werden noch verstärkt durch die Nationalisierungspolitik, die immer deutlicher auf den Kurs einer bürokratisierten, staatskapitalistischen, zentralverwalteten Wirtschaft hinausläuft. Der Staat ist seiner Aufgabe als Motor der wirtschaftlichen Entwicklung nicht gerecht geworden - eine Tatsache, die nur überraschen kann, wenn man diese Frage ideologisch betrachtet.

Aufgrund dieser krisenhaften Zuspitzung der wirtschaftlichen Situation sieht sich die Regierung zunehmend gezwungen, Dämme gegen mögliche politische Auswirkungen der Unzufriedenheit der Bevölkerung aufzubauen und mit repressiven Maßnahmen eine Opposition, die - wie gesagt - nur außerhalb des Parlamentes wirksam werden kann, im Keime zu ersticken.

In diesem Zusammenhang sind die Verfassungsänderung zur Einführung von Notstandsgesetzen ${ }^{25}$ und die Verabschiedung eines neuen Pressegesetzes, das der Regierung Mittel zur Beschränkung der Pressefreiheit in die Hand gibt ${ }^{26}$, zu nennen.

Die Inkonsequenz der Regierung bei der Durchführung politischer Entscheidungen wurde nach den Fehlleistungen im ökonomischen Bereich erneut offenkundig, als im April 1974 Mujibur Rahman den Notstand ausrufen, sich mit Sondervollmachten ausstatten ${ }^{27}$ und sogar die Streitkräfte mobilisieren ließ, um gegen das Bandenunwesen, gegen Schmuggler und gegen Wucherer vorzugehen. Diese Aktionen stießen bei der Mehrheit der Bevölkerung zunächst auf Zustimmung. Als sich indessen herauszustellen begann, daß die Ordnungshüter immer dann gestoppt wurden, wenn sie einflußreichen Mitgliedern der Awami-Liga auf die Spur gekommen waren, während Gegner der Regierung oft unter nichtigen Vorwänden inhaftiert wurden ${ }^{28}$, machte sich erneut Enttäuschung breit.

In den ersten beiden Jahren nach der erreichten Unabhängigkeit hatte die Kritik an der neuen Staatsführung vor "Bangabandhu“ - dem "Freund der Bengalen“ Halt gemacht. Diese "Schonfrist" scheint nunmehr abgelaufen zu sein. Man wirft ihm offen vor, aus falsch verstandener Loyalität seinen früheren Mitstreitern gegenüber Korruption und Unfähigkeit zu tolerieren ${ }^{29}$.

Mujibur Rahman geht nun seinerseits immer strenger gegen innerhalb und außerhalb seiner Partei stehende Opponenten vor. So ist fast der gesamte Parteivorstand der JSD ins Gefängnis gewandert oder aber ist, als sich die Verhaftungswelle ausbreitete, in den Untergrund gegangen, in dem seit langem die „Ostbengalische Befreiungsarmee" des Linksextremisten Siraj Sikdhar operiert ${ }^{30}$. Auch Maulani Bashani wurde unter Hausarrest gestellt, Studentenführer wurden aufgegriffen und regierungsfeindliche Mitglieder der Verwaltung ausgebootet. Es hat sich so ein Gefühl der politischen Verunsicherung ausgebreitet, das auch dem Ausländer nicht verborgen bleiben kann - sofern er in Dacca das Hotel Intercontinental als monumentalen Schutzhort verläßt und sich dorthin begibt, wo das eigentliche Bangladesh beginnt.

25 Vgl. dazu die Veröffentlichung des Textes in: The Bangladesh Gazette, 22. Sept. 1973, S. 6937 ff.

26 Siehe: The Bangladesh Gazette, 22. Sept. 1973, S. $6941 \mathrm{ff}$.

27 Dazu gibt das Sonderermächtigungsgesetz vom 5. 2. 1974 Handhabe, das von der Opposition heftigst kritisiert wurde. Siehe: The Bangladesh Observer, 6. 2. 1974.

28 Diese Aussage trifft Verf. aufgrund der in Bangladesh geführten Interviews und Nachforschungen. So auch: Werner Adam, Der Freiheitsheld hat seinen Glanz verloren, in: Frankfurter Allgemeine,

29 So ist in Bangladesh immer wieder der Fall des Präsidenten des Nationalen Roten Kreuzes, Ghazi Gulam Mustafa, zu hören, der an Skrupellosigkeit nicht zu übertreffen ist und es innerhalb von $z$ wei Jahren zum Besitz von weit über 30 Apotheken gebracht hat. Gleichwohl wird er vom Sheikh gedeckt, weil er Parteiboß der Awami-Liga im Distrikt Dacca ist, die ihm einen fast schrankenlosen Einfluß auf die Regierung sichert. - Darüber und über die Korruption im allgemeinen berichtete ausführlich: Carlos Widmann, Im großen Regen blüht die Korruption, in: Süddeutsche Zeitung, München, 27. Aug. 1974.

30 Siehe: Neue Zürcher Zeitung, 4. Sept. 1974 („Kritik an Sheikh Mujib in Bangladesh“). 
Diese einseitig gegen Regierungsopponenten gerichtete Kampagne fand ihr Gegenstück in einer von Mujibur Rahman eingeleiteten Kabinettsumbildung im Juli 1974. Im Zuge dieser Maßnahme wurden nicht weniger als neun Minister entlassen und zugleich eine Machtkonzentration eingeleitet, die in keiner Weise einer rationalen Handlungsweise der Regierung förderlich sein kann. Die Neu- und Umverteilung der Ressorts ${ }^{31}$ läßt eher eine Ưberlastung der einzelnen Minister als eine Verbesserung der Leistungsfähigkeit der Exekutiven erwarten. Doch offenkundig handelt es sich hier ohnehin nur um eine Übergangslösung mit dem Ziel, mehr Macht an sich zu ziehen und Widerstreiter zu eliminieren ${ }^{32}$. In diese Richtung deutet auch der im Oktober 1974 erzwungene Rücktritt des Finanzministers Tajuddin Ahmeds ${ }^{33}$, der politisch nach Moskau ausgerichtet ist und sich durch seine Kritik an den korruptiven Auswüchsen in Bangladesh den Unwillen des Regierungschefs auf sich gezogen hatte.

\section{Ausblick}

Von Anbeginn war die Demokratie in Bangladesh mehr formal als real gegeben. Es wird nun erkennbar, daß sich die Regierung Sheikh Mujiburs noch wesentlich autoritärer gibt als es die Dominanz der Partei erwarten lassen könnte. Daß das Bekenntnis zur Demokratie in den Hintergrund gestellt wird, kommt darin zum Ausdruck, daß im Parlament mehrere Gesetze zur Einschränkung der Grundrechte verabschiedet und diese konsequent zur Einschüchterung der Opposition eingesetzt wurden.

Diese Entwicklung läuft in Richtung auf ein Einparteien-Regime mit einhergehender Aushöhlung des demokratischen Verfassungsrahmen. Politischer Spielraum bleibt nur noch für die schon an die Kette gelegten NAP (M) und die CPB, mit denen die Awami-Liga bereits im November 1973 ein Aktionsprogramm unterzeichnete, um gemeinsam gegen „Hausierer, Schwarzmarkthändler, Schmuggler und asoziale Elemente" einzuschreiten ${ }^{34}$. Als Preis für die sowjetische Handels- und Militärhilfe müssen diese beiden Parteien toleriert werden. Die hieraus erwachsenen Bindungen für die Außenpolitik von Bangladesh dürften durch innenpolitische Einflußmöglichkeiten, die durch die Verbindung dieser Parteien mit der AwamiLiga geschaffen wurden, verstärkt werden. Möglicherweise versucht die Sowjetunion, einen ideologischen Stützpunkt für den „Marsch durch die parlamentarischen Institutionen" zu bilden - eine Taktik, die man auch in Indien eingeschlagen hat ${ }^{35}$. Jedenfalls dürfte es der Awami-Liga kaum erspart bleiben, sich der Unterstützung der moskautreuen „Mitarbeiter“ zu verschließen, wenn man allein der innerpolitischen Krise nicht gewachsen ist.

Gegenwärtig scheint man in Bangladesh weiter denn je von der Verwirklichung des indischen Modells entfernt zu sein und schon auf Gegenkurs zu gehen. Wohin der Weg allerdings führen wird, erscheint ungewiß. In informierten Kreisen von Dacca wird zwar von einer bevorstehenden Verfassungsänderung gesprochen.

31 Siehe: Frankfurter Allgemeine, 12. Juli 1974 („Mujibur Rahman sammelt die Macht um sich“).

32 In diesem Zusammenhang ist interessant, daß Konflikte im Kabinett nicht nach außen getragen werden, sie unbekannt bleiben und nicht nachgewiesen werden können, obwohl gewiß ist, daß zwischen den mehr Moskau, Delhi oder Washington zugeneigten Mitgliedern nicht immer Einigkeit bestehen kann.

33 Neue Zürcher Zeitung, 28. Okt. 1974.

34 Vgl.: Manifesto of People's United Front: Bangladesh, Dacca (o. J.).

35 In Indien paktieren die moskauhörigen Kommunisten mit der Kongreßpartei Indira Gandhis. 
Aber das sind spekulative Erwägungen, auch wenn manches darauf hindeutet, daß Mujibur Rahman seine persönliche Autorität noch stärker verankern möchte und dazu möglicherweise in einem Präsidialsystem eine Lösung sieht. Vor dem Hintergrund wachsender politischer Unrast und krisenhafter Wirtschaftsprobleme wäre dieses aber wohl nur bei gleichzeitig weiterer Einschränkung der demokratischen Rechte zu realisieren.

Aus der Sicht der Anhänger des demokratischen Verfassungsstaates ist die politische Entwicklung in Bangladesh enttäuschend. Aber an dem Beispiel von Bangladesh wird einmal mehr bestätigt, daß auch die „beste“ Verfassung ein Defizit der Regierung an Effizienz, Integrität und Rationalität nicht ausgleichen kann. Im Vergleich dazu wirkt Indien wirtschaftlich geradezu blühend, rational und relativ ehrlich verwaltet, obwohl die Schwächen der politischen Struktur auch hier offenkundig sind. Das aber stützt unsere Ansicht, daß weniger die auf dem indischen Modell basierende Verfassung im Hinblick auf die zu meisternden Probleme für Bangladesh inadäquat ist. In diesem Land sind es vielmehr die politischen Führungskräfte, die diese Verfassung ausfüllen und aus Ignoranz um ihre Verantwortung ihren Anteil am Versagen der Institutionen haben. 
Thought", followed by Party law which has precedence over constitutional law. This is revealed, with great clarity, in Article 2.

The crucial point of the revised Constitution, however, is the idea of "permanent revolution" on which the whole text is based. Not only have the authors emphasized the necessity of class struggle (against all enemies at home and abroad) but they also use "cultural revolutionary" language, referring to "great debates", "big-character posters", cadres participating in productive work etc., and encourage the permanent struggle against bureaucratic tendencies by simplifying the administration, reducing the codified rules of law in favour of the application of the mass line, etc.

\section{Parliamentary Democracy in Bangladesh - An Analysis of Constitutional State}

\section{By Horst Hartmann}

Bangladesh is one of the very few developing nations which have opted for parliamentary democracy after Independence. Obviously, the constitution-makers were influenced by the Indian "model" of a one-party-dominant-system, as they tried to stabilize the system by introducing special provisions against the desintegrative effects of partydissensions. However, it appears that the direction of constitutional development in Bangladesh is quite different from what was envisaged.

Both the law and order situation and economic conditions in Bangladesh have been very grave since it came into being. It is not for want of either legislative backing or administrative power that the government failed to cope with the threats to stability. As Prime Minister Sheikh Mujibur Rahman enjoys the unqualified support of the Awami League which in turn commands an overwhelming majority in the Parliament (Jayio Sangsad). There is no Opposition party able to provide a credible alternative national government. Indeed, the parliamentary Opposition is virtually dead - in contrast to the Indian situation. Last June the Government even enacted an amendment to the Special Powers Act authorising the government to arrest hoarders, blackmarketeers and smugglers. Hence, the Sheikh could not possibly claim that he lackes the power to restore economic distress and enforce law and order. On all accounts, the Sheikh did not act with determination and speed to tone up the administration and to combat starvation.

Sheik Mujibur Rahman has been Bangladesh's principal hope for stability and progress. However, as he has not produced results enough, the feeling of hopelessness and dispair has undermined his formerly undisputed Charisma. The dismissal of hundreds of government servants on charges of corruption in recent months is not known to have improved matters. The Sheikh has had reason to be disgusted with the politicians and their hangers-on who without doubt have abused their position to amass fortunes. But he has failed to exercise a healthy check on mismanagement and corruption, while using his special powers to repress opposition.

In fact, parliamentary democracy in Bangladesh was more formal than real from the very beginning of its existence. As it appears the disguised personal rule may 
be changed to the Presidential form of government. No doubt, there is hardly any indication that the Indian "model" will be realized.

The development in Bangladesh proves that even the "best" constitution can not compensate for a deficit of efficiency, integrity, and rationality on Government's side.

\section{Human Rights in Islamic View}

\section{By Udo STEINBACH}

The conception of inalienable and prenational existing human rights, bestowed upon the individual person "by nature" has emerged from the specific framework of occidental culture. It presupposes the conviction of the individual person's autonomous existence. Islam has not been able to do this. According to Islamic reading, rooted in the indissoluble unity of religion and political existence in this world, the Islamic community, through its actions, bears witness to God's rule, who governs it directly through His law (shari'a). Thus, the Islamic state, being a manifestation of God in this world, is the superior order to the interest of which every individual must submit itself; the individual person has a value only in so far as he takes part in pursuing the goals and missions of the Islamic Community.

In view of the significance accorded to the state by Islam, individual rights existing "per se" were not conceived. Although Islamic lawyers have worked out a system of rules, according to which the individual person was able to create for himself a humane existence, the continuity of the national order was more important to them than preserving the individual person's fundamental rights. And since through the passage of Islamic history, particularly since the 10th century, the power of the state was converted into a tyranny by the rulers, the individual person increasingly became a subordinate, lacking will and rights.

Islamic modernists and apoligists have tried to show the existence of human rights, in the occidental meaning, in Islam and have tried to embody the equality of all people, freedom of religion, personal liberty, and the right to have life and honor respected. Especially the 19th and 20th century reform of the Islamic world in regard to the political principles of the Occident has made human rights a constituent of the constitutions of nearly every Islamic country. However, it is still obvious that elements of a traditional system are impeding a total recognition of human rights. In particular the embodiment in the constitutions of Islam being the state religion indicates that even today the priority of terrestrial order lies less with the individual person bearing inalienable rights than in the realization of God's will. 